\title{
Sweet Excitements on Ginger Exosomal Therapeutics!
}

\section{Balasubramanyam*}

Dean of Research Studies and Senior Scientist, Madras Diabetes Research Foundation,

Chennai, India

*Corresponding Author: M Balasubramanyam, Dean of Research Studies and Senior Scientist, Madras Diabetes Research Foundation, Chennai, India.

DOI: $10.31080 /$ ASNH.2020.04.0660

\section{Ginger - the great medicine and the universal cure}

Ginger is revered in Ayurvedic medicine, where it is known as 'mahaoushadha' (the Great medicine) and as 'vishwabheshaj' or 'vishwaaushadha' (the Universal medicine). Since antiquity, ginger or Zingiber officinale, has been used by humans all over the world for medicinal purposes as well as spice condiments to enhance flavor in cooking. While ginger is rich in various chemical constituents, including phenolic compounds, terpenes, polysaccharides, lipids, organic acids, and raw fibers the health benefits of ginger are mainly attributed to its phenolic compounds, such as gingerol and shogaol and paradol.

\section{Modern science endorses health benefits of ginger}

Emerging literature demonstrate that ginger possesses multiple biological activities, including antioxidant, anti-inflammatory, antimicrobial, anticancer, neuroprotective, cardio and respiratory proective, antiobesity, antidiabetic, antinausea, and antiemetic activities [1]. Role of ginger and its constituents in ameliorating several disease-states has somewhat been thoroughly reviewed [2,3].

Recent studies imply that nanoparticles capable of colitis tissue-targeted delivery and site-specific drug release may become a novel therapeutic strategy. In this context, oral delivery of nanoparticles loaded with 6-Shogaol (an active ingredient of ginger), has been shown to attenuate ulcerative colitis and promote wound healing in a mice model [4]. Ginger and its bioactive compounds have been shown to have potential for cancer therapy because of their cytotoxic effect against cancer cell lines, combination therapy with chemotherapeutic and phenolic compounds, microbiome alterations as well as their use in nano-formulations [5]. While altered mitochondrial biogenesis and defective AMPK-PGC1 $\alpha$ signaling are considered major defects in diabetes and other metabolic disorders, recently it has been shown that 6-Gingerol (the major active component of ginger) promoted mitochondrial biogenesis and activated AMPK-PGC1 $\alpha$ signaling [6].

\section{Excitements on ginger exosomal therapy}

Exosomes are membrane-bound organelles generally secreted by eukaryotic cells that contain mRNAs, microRNAs, and/or proteins. However, recent studies have reported the isolation of these particles from foods and referred to as food-derived exosomes (FDEs) [7]. As they have a structure closely related to mammalian exosomes, they have been often called 'exosome-like nanovesicles' or 'exosome-like nanoparticles' (ELNs). Mu., et al. [8] have showed that ELNs isolated from four edible plants (ginger, carrot, grape and grapefruit) were taken up by intestinal macrophages and stem cells and exerted biological effects acting on different intracellular targets. They have also demonstrated among the other tested edible plant products, only ginger ELNs were mechanistically able to induce the gene expression of heme oxygenase-1(antioxidant) and cytokine IL-10 (anti-inflammatory). Compared to mammalian cell culture medium, plants are an advantageous source to scale up overall exosome yield. Several studies have demonstrated that exosome-like vesicles from edible plants including ginger can be used for therapeutic or delivery purposes [9].

While intestinal inflammation is characterized by defects in intestinal epithelial barrier function, abnormal immune responses, and altered diversity of gut microbiota, current agents used to treat inflammatory disorders have limitations. Sung., et al. [10] have recently purified and characterized ginger-derived nanoparticles (GDNPs) with anti-inflammatory activities and it has been claimed to target the inflamed intestinal mucosa, block damaging factors while promoting pro-healing factors. Working on nine food products that were selected to extract exosome-like nanoparticles (ELNs), Chen., et al. [11] have demonstrated ELNs from ginger rhizomes (G-ELNs) as new potent agents that inhibit NLRP3 (nucleotide-binding domain and leucine-rich repeat-containing family, pyrin domain-containing 3) inflammasome assembly and activation. It is suggested that the unique features of G-ELNs including biomolecule protection and tissue bioavailability should facilitate the development of G-ELN-based therapy to target the NLRP3 inflammasome in the disease settings. Although it is known that foods, including edible plants, are the main carbon and energy source for gut microbes, there is paucity of information on whether the expression of gut bacterial genes is affected by edible plant RNA. Teng., et al. [12] have showed that ginger ELNS (G-ELNs) are preferentially taken up by gut bacteria, resulting in changes in the composition and localization of bacteria, as well as in host physiology. This study has mechanistically demonstrated that certain specific miRNAs from G-ELNs targeted various genes in Lactobacillus rhamnosus (LGG) and notably enhanced gut barrier function to alleviate colitis. In another study, Sundaram., et al. [13] have showed that G-ELNs are selectively taken up by the periodontal pathogen Porphyromonas gingivalis in a G-ELN phosphatidic acid (PA) depen- 
dent manner via miRNA interactions with hemin-binding protein 35 (HBP35) and inhibit pathogenicity of Porphyromonas gingivalis.

With unique features of biomolecule protection and tissue bioavailability, G-ELNs represent a new class of therapeutics with high potential for translational clinical applications. With the everincreasing therapeutic values of ginger, it appears that Ginger becomes more of a sweet option for disease prevention as well as disease management.

\section{Bibliography}

1. Mao QQ., et al. "Bioactive Compounds and Bioactivities of Ginger (Zingiber officinale Roscoe)”. Foods 8.6 (2019). pii: E185.

2. Mohd Yusof YA. "Gingerol and Its Role in Chronic Diseases". Advances in Experimental Medicine and Biology 929 (2016): 177-207.

3. Mohd Sahardi NFN and Makpol S. "Ginger (Zingiber officinale Roscoe) in the Prevention of Ageing and Degenerative Diseases: Review of Current Evidence". Evidence-Based Complementary and Alternative Medicine (2019): 5054395.

4. Zhang M., et al. "Oral Delivery of Nanoparticles Loaded With Ginger Active Compound, 6-Shogaol, Attenuates Ulcerative Colitis and Promotes Wound Healing in a Murine Model of Ulcerative Colitis". Journal of Crohn's and Colitis 12.2 (2018): 217-229.

5. Mahomoodally MF, et al. "Ginger and its active compounds in cancer therapy: From folk uses to nano-therapeutic applications". Seminar on Cancer Biology (2019): pii: S1044-579X (19)30213-5.

6. Deng X., et al. "Promotion of Mitochondrial Biogenesis via Activation of AMPK-PGC1a Signaling Pathway by Ginger (Zingiber officinale Roscoe) Extract, and Its Major Active Component 6-Gingerol". Journal of Food Science 84.8 (2019): 2101-2111.

7. Munir J., et al. "Exosomes in Food: Health Benefits and Clinical Relevance in Diseases". Advances in Nutrition (2019): pii: nmz123.

8. $\mathrm{Mu} \mathrm{J.,} \mathrm{et} \mathrm{al.} \mathrm{"Interspecies} \mathrm{communication} \mathrm{between} \mathrm{plant} \mathrm{and}$ mouse gut host cells through edible plant derived exosomelike nanoparticles". Molecular Nutrition and Food Research 58 (2014): 1561-1573.

9. Zhuang X., et al. “Ginger-derived nanoparticles protect against alcohol-induced liver damage”. Journal of Extracellular Vesicles 4 (2015): 28713.

10. Sung J., et al. "Isolation, Purification, and Characterization of Ginger-derived Nanoparticles (GDNPs) from Ginger, Rhizome of Zingiber officinale”. Bio Protocol 9.19 (2019). pii: e3390.
11. Chen X., et al. "Exosome-like Nanoparticles from Ginger Rhizomes Inhibited NLRP3 Inflammasome Activation”. Molecular Pharmaceutics 16.6 (2019): 2690-2699.

12. Teng Y., et al. "Plant-Derived Exosomal MicroRNAs Shape the Gut Microbiota". Cell Host and Microbe 24.5 (2018): 637-652. e8.

13. Sundaram K., et al. "Plant-Derived Exosomal Nanoparticles Inhibit Pathogenicity of Porphyromonas gingivalis". iScience 21 (2019): 308-327.

\section{Assets from publication with us}

- Prompt Acknowledgement after receiving the article

- Thorough Double blinded peer review

- Rapid Publication

- Issue of Publication Certificate

- High visibility of your Published work

Website: https://www.actascientific.com/

Submit Article: https://www.actascientific.com/submission.php Email us: editor@actascientific.com Contact us: +919182824667 